\title{
Single-Atom Tip as an Emitter of Gas Field Ion Sources
}

\author{
W.-T. Chang ${ }^{1}$, I.-S. Hwang ${ }^{1}$, H.-S. Kuo ${ }^{1}$, T.-Y. Fu ${ }^{2}$, J.-L. Hou ${ }^{12}$,C.-Y. Lin $^{13}$ and T.-T. Tsong ${ }^{1}$ \\ 1. Institute of Physics, Academia Sinica, Taipei, Taiwan. \\ 2. Department of Physics, National Taiwan Normal University, Taipei, Taiwan. \\ 3. Department of Physics, National Taiwan University, Taipei, Taiwan.
}

Choice of emission of a fully coherent electron beam [1] or a high-brightness gas field ion beam [2] can be achieved easily with a single-atom tip (SAT) by switching the polarity of the emitter. The gas field ion beams emitting from Ir/W(111) SATs are not limited to helium and neon. They also include reactive gases such as hydrogen, oxygen and nitrogen [3-4]. Thus, the functions of scanning ion microscopy, secondary-ion mass spectrometry (SIMS), electron microscopy and nano-fabrication may be integrated within one single instrument, as shown in Fig. 1.

We have demonstrated hydrogen, helium, argon, and oxygen ion beams emitting from a Ir/W SAT and characterized these ion sources. Fig.2 shows the current stability of three different gas ion beams emitted from a Ir/W SAT. Clearly, they are very stable with instability (standard deviation/mean current) of 3\% for the $\mathrm{He}^{+}$ion beam, $5 \%$ for the $\mathrm{H}^{2+}$ ion beam, and $7.7 \%$ for the $\mathrm{O}^{2+}$ ion beam [3]. Hydrogen and helium ions provide the lowest sputtering rates, which is beneficial for scanning ion microscopy as well as for fabrication of nanostructures of high aspect ratios. The argon ion has a large mass and can provide a high sputtering rate, suitable for ion milling. Due to the high secondary ion yields, an oxygen ion beam may be applied to nano-SIMS. The ion beam profiles indicate that the half opening angle is $\sim 0.5^{\circ}$ or smaller. This single spot indicates that emission occurs only from the topmost atom. The smallest source size and the small opening angle are particularly favorable for achieving high angular intensity, high brightness, and low spherical aberration, which are important characteristics for a focused ion beam system. The GFIS emitted from a single-atom tip is also highly coherent and may make possible precision measurements such as ion interferometry [5]. The current of these gas ion beams are very stable and the tip does not show any degradation under fields above $5 \mathrm{~V} / \AA$ after a total operation time of 80 hours. Since the SAT can be regenerated by annealing for more than 50 times, therefore its lifetime is long enough for most practical applications.

In addition, we have also prepared a pure iridium SAT based on oxygen-induced crystal faceting of the $\operatorname{Ir}(210)$. Fig. 3 shows the current stability of four different gas ion beams emitted from an Ir-SAT. Obviously, they are very stable with instability below $5 \%$ [4]. Thus the Ir-SAT can be a good field ion emitter, capable of emitting a variety of gas ion beams, including $\mathrm{He}^{+}, \mathrm{H}^{2+}, \mathrm{N}^{2+}$, and $\mathrm{O}^{2+}$, with high brightness. In particular, nitrogen is a very corrosive gas for most metal tips, including pure tungsten tips, under strong positive electric fields. This suggests that many other gas field ion beams may also be emitted from this Ir-SAT and can greatly broaden the application of focused ion beam technology.

References:

[1] C.-C. Chang, Nanotechnology 20 (2009) 115401.

[2] H.-S. Kuo et al., Nano Lett. 4 (2004), 2379.

[3] H.-S. Kuo et al., Appl. Phys. Lett. 92 (2008), 063106.

[4] H.-S. Kuo et al., Nanotechnology 20 (2009), 335701.

[5] F. Hasselbach, Rep. Prog. Phys. 73 (2010) 016101. 


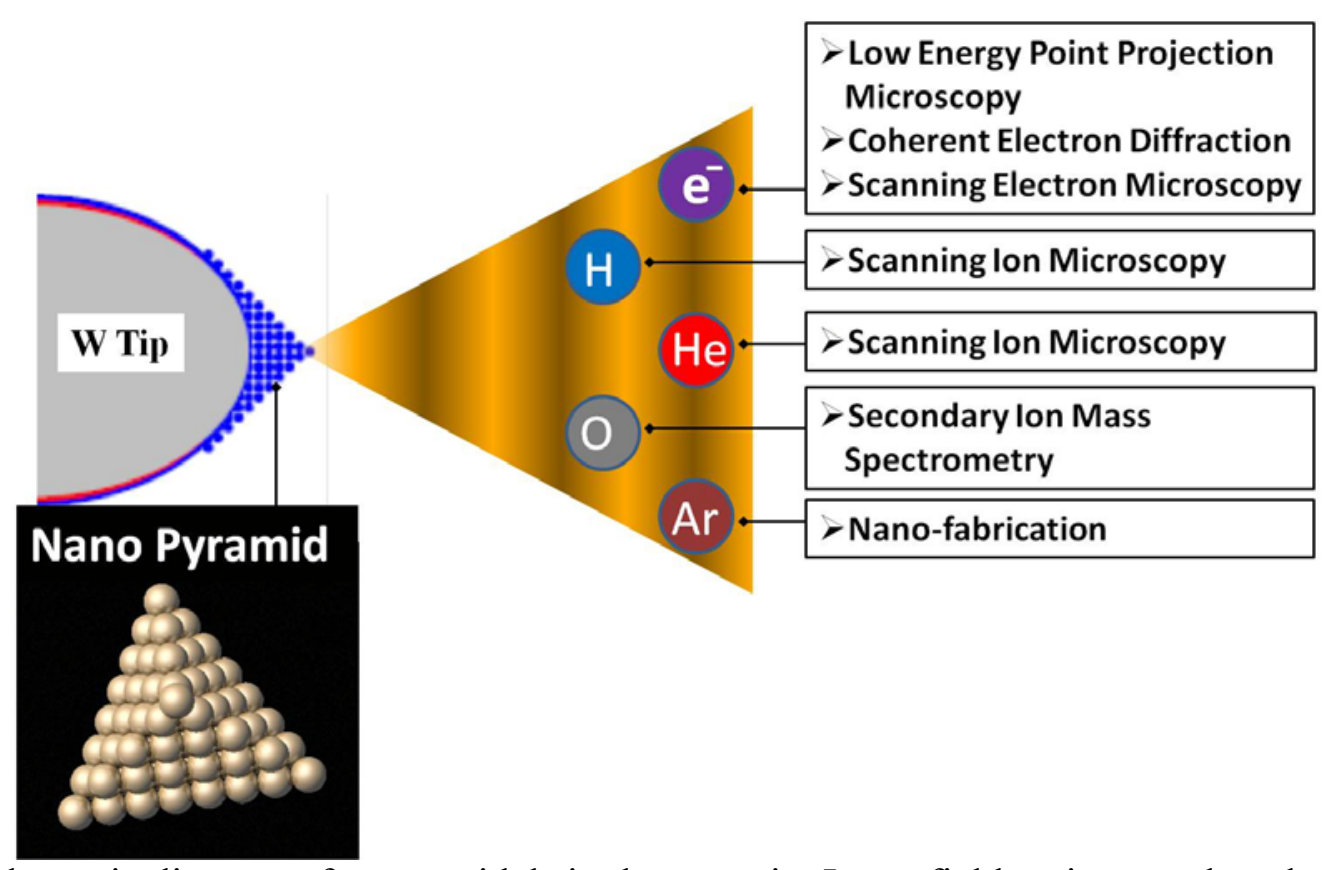

Figure 1. Schematic diagram of a pyramidal single-atom tip. It can field emit not only coherent electron beam but also various ion beams for different applications.

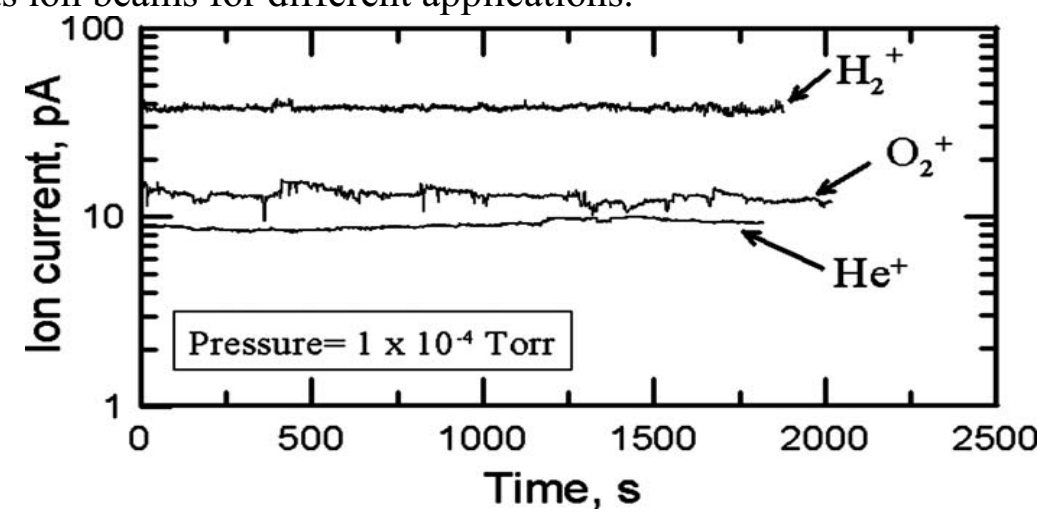

Figure 2. Current stability of three different gas ion beams emitted from a Ir/W SAT.

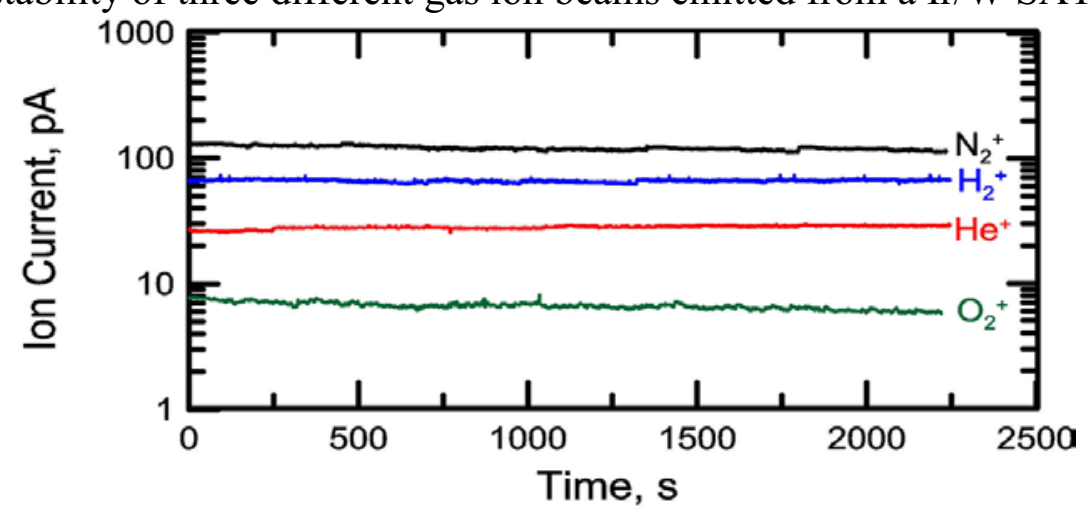

Figure 3. Current stability of four different gas ion beams emitted from an Ir-SAT 